\title{
Emprego de metilfenidato para o tratamento de déficit cognitivo em paciente com seqüela de traumatismo cranioencefálico
}

\author{
Methylphenidate for the treatment of cognitive deficit in traumatic brain injury
}

\author{
Fábio Lopes Rocha, Leandro F. Malloy-Diniz e Cláudia Hara
}

\begin{abstract}
Resumo
O traumatismo craniencefálico (TCE) é a principal causa de morte e deficiência em jovens. Déficits da atenção e das funções executivas são freqüentemente relatados após comprometimentos do córtex préfrontal. Os autores relatam um caso de emprego do metilfenidato para o tratamento de alterações cognitivas em paciente com cerca de dois anos de evolução pós-TCE. Com dois meses de tratamento, o paciente relatou melhora significativa de suas dificuldades cognitivas, com maior poder de concentração na leitura, melhor capacidade de manter a atenção em conversas e filmes, além de redução do número de vezes em que perdia objetos. Como efeito colateral, houve um pequeno aumento da irritabilidade nas primeiras duas semanas de tratamento. Ao exame neuropsicológico, constatou-se melhora substancial nas medidas de velocidade de processamento, nos erros por omissão e nos erros por comissão.

Palavras-chave: metilfenidato, traumatismo craniencefálico, déficit cognitivo, avaliação neuropsicológica.
\end{abstract}

\begin{abstract}
Traumatic brain injury is the main cause of death and disability among young people. Attention deficits and executive dysfunction occur frequently after prefrontal cortex damage. The authors report a case of methylphenidate use for the treatment of cognitive deficits in a patient with a two-year evolution of traumatic brain injury. After two months, the patient reported significant improvement in his cognitive deficits, with increased ability to pay attention to reading, talking, watching films and reduction in the frequency of losing objects. As a side effect, he complained of a small increase in irritability in the first two weeks. The neuropsychological assessment showed a substantial improvement in the mental processing speed, in the omission errors and in the commission errors.

Key words: methylphenidate, traumatic brain injury, cognitive deficit, neuropsychological evaluation.
\end{abstract}

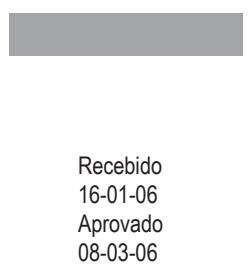

Instituto de Previdência dos Servidores do Estado de Minas Gerais (IPSEMG) (Rocha FL, Hara C) Faculdade de Ciências da Saúde da Universidade Fundação Mineira de Educação e Cultura (FUMEC); Sociedade Mineira de Neuropsicologia (SMN) (Malloy-Diniz, LF) Faculdade de Saúde e Ecologia Humana (FASEH) (Hara C)

Correspondência para: Fábio Lopes Rocha

Rua dos Otoni, 106 - Santa Efigênia - 30150-270 - Belo Horizonte-MG - Telefax: (31) 3241-1474

- e-mail: rochafl@uol.com.br 


\section{Introdução}

O traumatismo craniencefálico (TCE) é a principal causa de morte e deficiência em jovens (Silver et al., 2004). Além das conseqüências neurológicas do TCE, os problemas comportamentais, cognitivos e sociais acarretam prejuízo significativo. A patofisiologia do TCE é um processo dinâmico de longo prazo. Os aspectos clínicos e fisiológicos do processo de recuperação podem se estender por anos (Grafman e Salazar, 1996). Além do dano cerebral direto resultante do trauma, ocorre uma série de eventos que contribuem para maior extensão dos danos neuronais. Por exemplo, rapidamente instala-se um desequilíbrio entre demanda e oferta de energia, isto é, paralelamente ao aumento da necessidade de energia pelos neurônios afetados há redução do fluxo sangüíneo cerebral. As células afetadas também são mais vulneráveis à hipoxia. Secundariamente, ocorrem alterações no fluxo de cálcio, processos inflamatórios são desencadeados, ocorre liberação de excitotoxina, há ativação de fosfolipase e de protease. Também ocorre liberação de radicais livres e de glutamato, que contribuem para 0 aumento do dano celular (Silver et al., 2004).

A patologia do TCE pode ser classificada em quatro componentes:

- lesão focal;

- lesão axonal difusa;

- hipoxia/isquemia superimposta;

- posteriormente, lesão microvascular difusa com perda da auto-regulação (Grafman e Salazar, 1996).

As lesões focais acarretam deficiências relacionadas ao local da lesão. A localização mais freqüente de contusões após aceleração/desaceleração são os lobos orbitofrontal e temporal anterior. As seqüelas clínicas mais importantes são anormalidades comportamentais e cognitivas relacionadas a essas regiões. A lesão axonal difusa, uma das causas mais importantes de déficit neurológico persistente grave, é decorrente de lesões mecânicas e/ou químicas de axônios na substância branca cerebral. A isquemia é um dos principais fatores determinantes da evolução clínica. O dano microvascular difuso é acompanhado de perda da auto-regulação cerebrovascular. Em seguida, ocorrem danos teciduais secundários. Eventos bioquímicos desencadeados pelo trauma podem, em última análise, ser o principal responsável pela perda tecidual cerebral (Silver et al., 2004).

O TCE é uma causa comum de anormalidades neuropsiquiátricas (Silver et al., 2004; Grafman e Salazar, 1996; Vakil, 2005). Alterações muito variadas podem ocorrer, mas tipicamente há comprometimento da personalidade e da cognição social, memória, atenção e funcionamento executivo. 0 paciente pode perder inibições sociais, comportar-se de forma irrefletida, ignorar convenções sociais e não avaliar adequadamente as conseqüências de seus atos. O TCE afeta uma ampla gama de aspectos da memória. Geralmente ocorrem amnésia pós-traumática, que pode persistir por semanas a meses, e amnésia retrógrada. As alterações de atenção são freqüentes no TCE, particularmente a redução da capacidade de concentração. $O$ paciente pode ter dificuldade em focalizar novamente a atenção após período de espera e em inibir respostas a estímulos previamente associados. Outros problemas freqüentemente encontrados em pacientes com TCE são a dificuldade em formação de conceitos e a perseveração. Pode ainda haver mudanças do humor, agressividade e violência, bem como transtornos afetivos e de ansiedade, psicose e transtornos do sono.

Vários medicamentos têm sido propostos para 0 tratamento dos déficits cognitivos dos pacientes com TCE, entre eles dextroanfetamina, metilfenidato, levodopa, bromocriptina, amantadina, antidepressivos tricíclicos, modafinil, atomoxetina, bupropiona, inibidores da acetilcolinesterase e memantina (Silver et al., 2004; Napolitano et al., 2005). Entretanto, os poucos estudos controlados têm pequeno número de pacientes, abrangem populações em diferentes etapas de evolução e, freqüentemente, não avaliam resultados em longo prazo (Silver et al., 2004).

Os autores deste artigo relatam um caso de emprego do metilfenidato para o tratamento de alterações cognitivas em paciente com cerca de dois anos de evolução pós-TCE.

\section{Relato de caso}

F., sexo masculino, universitário, sofreu acidente automobilístico aos 23 anos, em junho de 2003. Foi internado com TCE, edema cerebral difuso e fratura dos ossos frontal esquerdo e da face. Esteve em coma por, aproximadamente, quatro semanas com diagnóstico de lesão axonal difusa grave. Recebeu alta com quadro de tetraparesia espástica e alterações cognitivas globais.

Dois meses após o acidente, a tomografia encefálica mostrou hipodensidade nas regiões frontal e temporal direitas e hipotrofia cortical frontal bilateral. Nessa época, a avaliação neuropsicológica evidenciou alterações comportamentais relacionadas às funções executivas, envolvendo dificuldades na auto-regulação comportamental, flexibilidade cognitiva e memória de trabalho. O paciente recebeu diagnóstico de transtornos de personalidade e comportamentais devidos à doença, lesão e disfunção cerebrais, segundo a Classificação Internacional de Doenças 10 ([CID-10] F07.8). Após quatro meses, o exame de ressonância magnética cerebral mostrou contusões múltiplas e micro-hemorragias secundárias à lesão axonal difusa.

Após um ano do acidente, familiares e o paciente relatavam períodos de heteroagressividade, alterações de comportamento e déficit de concentração. Em nova avaliação neuropsicológica, evidenciaram-se alterações de memória, déficit de concentração, alterações de linguagem e lentificações motora e do pensamento.

Em tratamentos psiquiátricos prévios, o paciente fez uso de diversos psicofármacos para controle de episódios depressivos e períodos de agressividade, particularmente fluoxetina, pipotiazina, tioridazina, olanzapina, quetiapina e risperidona, além de benzodiazepínicos.

Em novembro de 2004, 17 meses após o TCE, em virtude da história de alteração de humor caracterizada por excitação, impulsividade, irritabilidade, explosividade, 
agressividade, logorréia e insônia, bem como episódios depressivos, foi aventada a hipótese de transtorno de humor orgânico (CID-10 F06.3) e iniciado tratamento com oxcarbazepina. Houve melhora significativa do quadro de humor. Persistiam, entretanto, as queixas relacionadas ao déficit cognitivo.

Em junho de 2005, dois anos após o acidente, o exame neuropsicológico revelou memória visuoespacial aquém dos limites da normalidade e dificuldades relacionadas ao efeito de distratores retroativos e proativos. As habilidades de memória e aprendizagem auditivo-verbal encontravam-se no limite inferior da normalidade. As tarefas que envolviam memória de trabalho, flexibilidade cognitiva, planejamento e solução de problemas encontravam-se abaixo dos limites da normalidade. Comparado ao exame realizado 22 meses antes, próximo ao acidente, constatou-se manutenção das dificuldades apresentadas pelo paciente em termos cognitivos.

Em agosto de 2005, iniciou-se metilfenidato na tentativa de melhorar os aspectos cognitivos do paciente. Em termos subjetivos, nos dois meses seguintes de tratamento, o paciente relatou melhora significativa de suas dificuldades cognitivas. Afirmou estar mais atento durante leituras, relatou melhora na capacidade de manter a atenção em conversas, pois antes se distraía com certa facilidade, percebeu redução no número de vezes em que perdia objetos e também uma melhor capacidade no acompanhamento de filmes. Relatou, como efeito colateral, pequeno aumento da irritabilidade nas primeiras duas semanas de tratamento.

Em termos objetivos, foi realizado novo exame neuropsicológico das funções de atenção sustentada e controle inibitório, tendo sido utilizado o paradigma Continuous Performance Test-II. O exame foi realizado em setembro de 2005, cerca de 40 dias após início do metilfenidato, e evidenciou melhora substancial nas medidas de velocidade de processamento, erros por omissão e por comissão (Figuras 1 e 2).

Figura 1. Comparação entre as medidas de tempo de reação no CPT-II nas avaliações anterior e posterior ao uso do metilfenidato

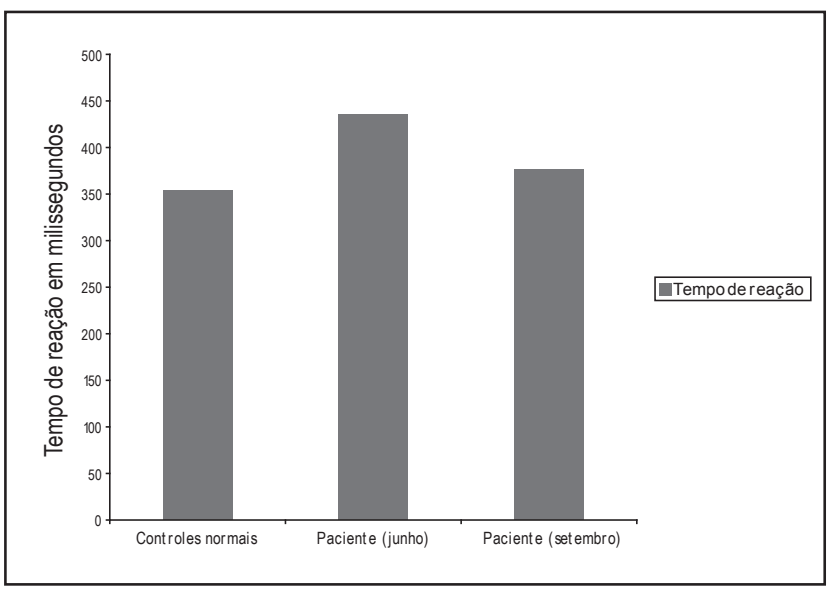

Figura 2. Comparação entre a quantidade de erros por omissão e comissão no CPT-II nas avaliações anterior e posterior ao uso do metilfenidato

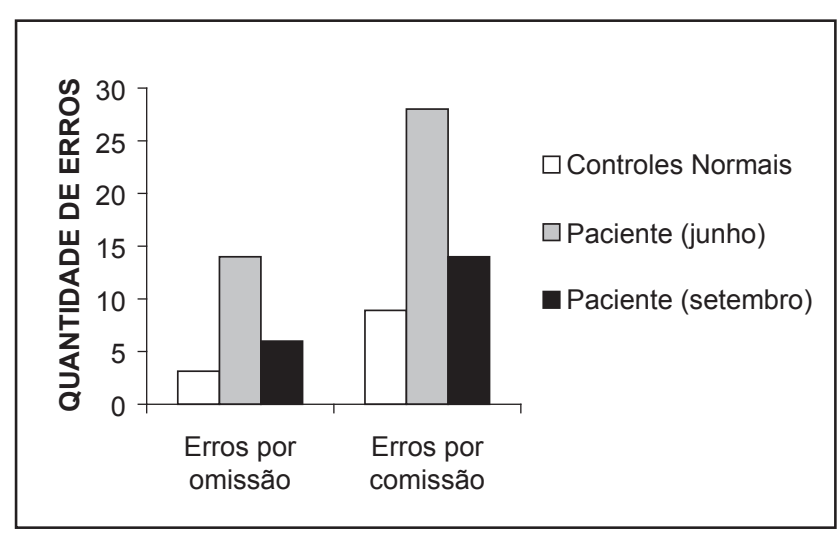

\section{Discussão}

Déficits de atenção e das funções executivas são freqüentemente relatados após comprometimentos do córtex pré-frontal. Pacientes com esse padrão de comprometimento geralmente apresentam dificuldades em tarefas que demandam organização, planejamento, solução de problemas, controle inibitório, flexibilidade cognitiva e memória de trabalho (Silver et al., 2004; Grafman e Salazar, 1996; Vakil, 2005). Essas deficiências cognitivas são observadas no dia-a-dia em prejuízos acadêmicos, profissionais, sociais (Lehtonen et al., 2005) e econômicos (Spinella et al., 2004), semelhantes aos apresentados pelo paciente.

O uso de metilfenidato mostrou-se útil na redução de déficits cognitivos resultantes de TCE, particularmente alterações da atenção, mesmo quando introduzido cerca de dois anos após 0 acidente.

Há vários relatos de casos sobre os benefícios do metilfenidato em pacientes com déficits cognitivos pós-TCE (Napolitano et al., 2005). Além disso, estudos controlados avaliaram a eficácia e a segurança do emprego desse medicamento no tratamento do déficit cognitivo e alterações de comportamento em crianças e adultos com TCE.

Em geral, os resultados sugerem a utilidade do metilfenidato na melhora de memória, atenção, concentração e velocidade do processamento mental, embora nem todos os aspectos sejam evidenciados ao mesmo tempo e em todos os estudos (Napolitano et al., 2005; Lee et al., 2005; Plenger et al.,1996; Whyte et al., 1997 e 2004). Além do mais, alguns estudos mostram resultados negativos (Speech et al., 1993; Mooney e Haas, 1993). Esses efeitos parecem ser independentes da atuação em sintomatologia depressiva associada (Lee et al., 2005).

O metilfenidato, geralmente, é bem tolerado. Os eventos adversos mais comuns são cefaléia, perda de apetite, epigastralgia, dispepsia, insônia, ansiedade e irritabilidade. No caso relatado, o medicamento foi muito bem tolerado, acarretando apenas leve irritabilidade no início do tratamento. Recomenda-se o tateamento da dose para avaliação do sur- 
gimento de efeitos adversos e avaliação da eficácia. Inicia-se com dose de 10 a $20 \mathrm{mg} / \mathrm{dia}$, em duas tomadas, com reajuste a cada três dias, no caso do metilfenidato de liberação imediata, ou com dose de $18-20 \mathrm{mg} / \mathrm{dia}$ e reajuste semanal, no caso de medicações de liberação controlada ou estendida. A dose máxima recomendada é de $60 \mathrm{mg} /$ dia de metilfenidato, podendo ser aumentada em situações especiais (Bezchlibnyk-Butler e Jeffries, 2005).

Pacientes com história de TCE têm risco aumentado para depressão maior, distimia, transtorno de pânico, transtorno obsessivo-compulsivo (TOC), transtorno fóbico e abuso/dependência de drogas (Silver et al., 2001). Há também relatos de mania pós-TCE (Burstein, 1993).

O paciente do presente relato de caso vinha em tratamento bem-sucedido de alterações do humor resultantes do TCE com o emprego de oxcarbazepina. Ressalta-se que a introdução do metilfenidato não provocou alterações na estabilidade do humor. Também não há relato de interação medicamentosa entre as duas substâncias (Bezchlibnyk-Butler e Jeffries, 2005).

Evidentemente fatores inespecíficos (evolução natural, efeito placebo, entre outros), impossíveis de serem controlados em um estudo de caso, podem ter sido os responsáveis pela meIhora do paciente. Entretanto, seria pouco provável a ocorrência de melhora aguda em um caso estável em relação aos déficits cognitivos há cerca de dois anos, conforme documentado por exames neuropsicológicos.

\section{Conclusão}

O presente relato também ilustra a importância das avaliações neuropsicológicas no acompanhamento clínico de pacientes com TCE, para documentar os déficits cognitivos e intelectuais, auxiliando no esclarecimento diagnóstico e no estabelecimento de condutas terapêuticas (Malloy-Diniz, 2001). Em síntese, este relato corrobora a literatura, sugerindo que o emprego de metilfenidato pode ser benéfico na redução de alterações cognitivas em pacientes com seqüela de TCE. São necessários ensaios clínicos controlados com placebo para determinação de dose ótima, melhor fase do TCE para se iniciar o tratamento, duração do tratamento e efeitos em longo prazo em pacientes com TCE leve, moderado ou grave.

\section{Referências}

Silver JM, Hales RE, Yudofsky SC. Neuropsychiatric aspects of traumatic brain injury in essentials of neuropsychiatric and clinical neurosciences. In: Yudofsky SC, Hales RE, editors. American Psychiatric Publishing, p. 241-91, Washington, DC, 2004.

Grafman J, Salazar A, Traumatic brain injury in neuropsychiatry. Fogel BS, Schiffer RB, Rao SM, editors. Williams \& Wilkins, p. 935-45, Baltimore, 1996.

Vakil E. The effect of moderate to severe traumatic brain injury (TBI) on different aspects of memory: a selective review. J Clin Exp Neuropsychol, 27(8): 977-1021, 2005.

Napolitano E, Elovic EP, Qureshi Al. Pharmacological stimulant treatment of neurocognitive and functional deficits after traumatic and nontraumatic brain injury. Med Sci Monit, 11(6): RA212-20, 2005

Lehtonen S, et al. Neuropsychological outcome and community reintegration following traumatic brain injury: the impact of frontal and non-frontal lesions. Brain Inj, 19(4): 239-56, 2005.

Spinella M, Bijou Y, Lester D. Prefrontal system dysfunction and credit card debt. International Journal of Neuroscience, 114(10): 1323-32, 2004

Lee $\mathrm{H}$, et al. Comparing effects of methylphenidate, sertraline and placebo on neuropsychiatric sequelae in patients with traumatic brain injury. Hum Psychopharmacol, 20(2): 97-104, 2005.
Plenger PM, et al. Subacute methylphenidate treatment for moderate to moderately severe traumatic brain injury: a preliminary double-blind placebo-controlled study. Arch Phys Med Rehabil, 77(6): 536-40, 1996.

Whyte $\mathrm{J}$, et al. Effects of methylphenidate on attention deficits after traumatic brain injury: a multidimensional, randomized, controlled trial. Am J Phys Med Rehabil, 83(6): 401-20, 2004.

Whyte $\mathrm{J}$, et al. Effects of methylphenidate on attentional function after traumatic brain injury. A randomized, placebo-controlled trial. Am J Phys Med Rehabil, 76(6): 440-50, 1997.

Speech TJ et al. A double-blind controlled study of methylphenidate treatment in closed head injury. Brain Inj, 7(4): 333-8, 1993.

Mooney GF, Haas LJ. Effect of methylphenidate on brain injury-related anger. Arch Phys Med Rehabil, 74(2): 153-60, 1993.

Bezchlibnyk-Butler KZ, Jeffries JJ. Clinical Handbook of Psychotropic Drugs. 15 ed. Ashland: Hogrefe \& Huber Publishers; 2005, p. 344.

Silver JM, et al. The association between head injuries and psychiatric disorders: findings from the New Haven NIMH Epidemiologic Catchment Area Study. Brain Inj, 15(11): 935-45, 2001.

Burstein A. Bipolar and pure mania disorders precipitated by head trauma. Psychosomatics, 34(2): 194-5, 1993.

Malloy-Diniz LF. 0 exame neuropsicológico e suas contribuições à Psiquiatria. Psiquiatria Biológica, 9(2): 66-77, 2001. 